\title{
Simulation of the energy spectra of swift light ion beams after traversing cylindrical targets: A consistent interpretation of experimental data relevant for hadron therapy ${ }^{\star}$
}

\author{
Pablo de Vera ${ }^{1, a}$, Rafael Garcia-Molina ${ }^{2}$, and Isabel Abril ${ }^{3}$ \\ 1 MBN Research Center, Altenhöferalle 3, 60438 Frankfurt am Main, Germany \\ 2 Departamento de Física - Centro de Investigación en Óptica y Nanofísica, Regional Campus of International Excellence \\ "Campus Mare Nostrum", Universidad de Murcia, 30100 Murcia, Spain \\ 3 Departament de Física Aplicada, Universitat d'Alacant, 03080 Alacant, Spain
}

Received 14 February 2019 / Received in final form 23 May 2019

Published online (Inserted Later)

(C) EDP Sciences / Società Italiana di Fisica / Springer-Verlag GmbH Germany, part of Springer Nature, 2019

\begin{abstract}
We have performed detailed simulations of the energy spectra, recorded at several angles, of proton and helium ion beams after traversing thin cylindrical targets of different nature (liquid water and ethanol jets, as well as a solid aluminium wire), in order to reproduce a series of measurements intended to assess the stopping power of $0.3-2 \mathrm{MeV}$ ions. The authors of these experiments derived values of the stopping power of liquid water (a quantity essential for the evaluation of radiation effects in materials, particularly for radiotherapy purposes) that are $\sim 10 \%$ lower than what is expected from other measurements and theories. In our simulations, instead of treating the stopping power as an unknown free parameter to be determined, we use as input the electronic stopping power accurately calculated within the dielectric formalism. We take into account in the simulations the different interactions that each projectile can experience when moving through the target, such as electronic stopping, nuclear scattering or electron charge-exchange processes. The detailed geometry of the target is also accounted for. We find that our simulated energy distributions are in excellent agreement with the published measurements when the diameter of the cylindrical targets is slightly reduced, what is compatible with the potential evaporation of the liquid jets. On the basis of such an excellent agreement, we validate the accuracy of the model we use to calculate electronic excitation cross sections for ions in condensed matter in its range of applicability (particularly the electronic stopping power) needed for charged particle transport models, and we offer a consistent, but alternative, interpretation for these experiments on ion irradiation of cylindrical targets.
\end{abstract}

\section{Introduction}

The energy loss of fast charged particles in condensed matter has a great interest due to its important applications in science, industry and medicine. In particular, the interaction of ion beams with biological materials is of great relevance for ion beam cancer therapy or hadron therapy as well as for radiation protection. The former is a promising technique for killing tumour cells, whose physics rationale lies in how ions move through a medium almost in ballistic trajectories (i.e., with small lateral dispersion), in the manner in which they deliver most of their energy at the end of their trajectories (in the so-called Bragg peak region) by means of electronic excitations, as well as

* Contribution to the Topical Issue "Dynamics of Systems on the Nanoscale", edited by I. Bald, I.A. Solov'yov, N.J. Mason and A.V. Solov'yov.

a e-mail: devera@mbnexplorer.com in the way in which ions interact with biomolecular targets on the nanometre scale, which enhances the effects of radiation $[1,2]$. Ion beams allow destroying tumours while preserving the healthy tissue, which is crucial for cancers in close vicinity to critical structures or sensitive organs. As for radiation protection, damage to human tissues by ions can happen as a result of natural radioactivity, accidental exposure at nuclear power plants or by radiation in space. The latter represents an important limiting factor for manned space missions [3]. Hence, it is a critical issue to accurately characterize the electronic interaction of ions with liquid water (the main constituent of living tissues) for radiotherapy and radiation protection purposes.

Ion beam damage of biomaterials is a complex problem, which involves phenomena in multiple space, time and energy scales $[2,4]$. It starts in a macroscopic scale with the energy loss of ion beams of tens or hundreds of $\mathrm{MeV} / \mathrm{u}$ moving through biological targets, which has a length scale of tens of centimetres. The next step consists 
on the production of secondary electrons in the biological targets. The characteristic length scale of this step is microscopic, since the produced secondary electrons, and the other tertiary electrons produced by them, have energies mainly of a few $\mathrm{eV}$, so they will travel distances of nanometres around the ion's path. These are, precisely, the characteristic length scales of the biological macromolecules, which are regarded as the sensitive targets in radiobiological models. Finally, this shower of electrons may produce clustered damage in these ultimate nanometric targets, such as DNA, proteins, or other biomolecules. The level of clustering of damage events in these nanometric targets will determine the final effects of radiation $[4,5]$.

Simulation of the transport of ion beams and secondary electrons in condensed-phase biotargets within arbitrary geometries, as well as their interaction with the relevant biomolecules, is often performed by means of Monte Carlo (MC) techniques [6]. A critical point for the reliability of MC simulations is the availability of accurate cross sections for all the possible processes that can occur in the interaction of radiation with matter. The interaction of charged particles with liquid water, the main constituent of biological media, is especially important. In the energy range of interest in hadron therapy (from several hundreds of $\mathrm{keV} / \mathrm{u}$ to several hundreds of $\mathrm{MeV} / \mathrm{u}$ ) the electronic interactions are the main cause of the slowing down of ion beams and of the production of secondary electrons, and thus to know the electronic cross sections of ions in liquid water and other biomaterials is of capital importance. It should be noted that, despite its intrinsic importance, experimental data for the electronic stopping power and other electronic cross sections in liquid water is scarce, due to the difficulties of doing measurements in the liquid phase.

Traditionally, electronic interaction cross sections for water vapour were used in Monte Carlo codes, being scaled to liquid water density, in order to simulate the biological medium, since most of the experimental and theoretical information was available for that target. Nowadays, several methodologies have been developed to calculate [7-13] and measure [14-18] different quantities for the energyloss of charged particles with liquid water, which is a much more realistic representation of biological targets. Particularly, the dielectric formalism [19] has been established over the last years as a reliable and convenient methodology for evaluating electronic cross sections, not only in liquid water, but also in other condensed-phase biological targets. Even though initially this method was mainly used to obtain total energy-loss quantities (such as the stopping power), it was latter demonstrated to be very useful to obtain other important quantities such as electronic excitation and ionization cross sections, both for ions $[9,11,20-22]$ and for electrons [13,20,23], which are a needed input for the multiscale simulation of charged particle beams interaction with biomaterials.

Still, the determination of the fundamental energy-loss quantities in liquid water, such as the electronic stopping power (one of the main basic electronic interaction quantities which, among other issues, determines the range of projectiles in tissue [24]), is an ongoing problem [25].
In recent times, two experiments concerning the stopping power of liquid water for proton beams have been reported. The one by the Kyoto group [14-17], for energies from $0.3-2 \mathrm{MeV}$, appears to be about $10 \%$ low with respect to current theories [26]. Whereas the other one by the Jyväskylä group [18], for proton energies from 4.8-15.2 MeV, agrees nicely with the Bethe theory and other theoretical calculations. This discrepancy can be understood from the form in which these stopping power data were obtained. The data by [18] where directly assessed by transmission through a thin liquid water foil (enclosed within two thin copper sheets). On the other hand, the data by the Kyoto group [17] were deduced from the analysis of the proton energy spectra emerging at different angles after traversing a liquid water jet by means of simulations with the Geant4 code [27]. The stopping power of liquid water and the diameter of the liquid jet were treated as fitting parameters to be determined in these simulations. A similar procedure was repeated for other cylindrical targets made of liquid ethanol $[16,28,29]$ and solid aluminium [16].

In this paper we use the SEICS code $[8,30]$ to simulate the experiments performed by the Kyoto group in the different targets, where the energy spectra of protons or alpha particles reaching the detector at several angles after traversing a cylindrical target (either a liquid water jet, a liquid ethanol jet or an aluminium wire) were reported. In all the cases, our simulations are fed with reliable electronic stopping powers obtained within the dielectric formalism. It is demonstrated that our simulations are able to perfectly reproduce the complete set of measurements in the three target materials by only using the diameter of the cylinder as a fitting parameter, in contrast to the results shown by the Kyoto group. We thus offer an alternative interpretation of their measurements and check the reliability of the dielectric formalism for calculating the electronic stopping power (as well as other electronic interaction quantities), so important for the description of the radiation damage of biomaterials.

This paper is organized as follows. A description of the experimental set-up developed by the Kyoto group, to be reproduced in our simulations, is presented in Section 2. The theoretical calculation, within the dielectric formalism, of the electronic stopping power of condensed targets for swift ions is outlined in Section 3. The transport of ions through condensed media is simulated by the SEICS code, which is described in Section 4, whereas the comparison with the experimental results and the corresponding discussion are presented in Section 5. Finally the conclusions of the work appear in Section 6.

\section{Description of the experimental set-up from Kyoto group}

The stopping power of ions in condensed matter is one of the main input parameters in MC simulations. However, measurements of the stopping power of protons in liquid water are scarce. Apart from the thin film measurements reported at high energies by [18], a previous experiment 


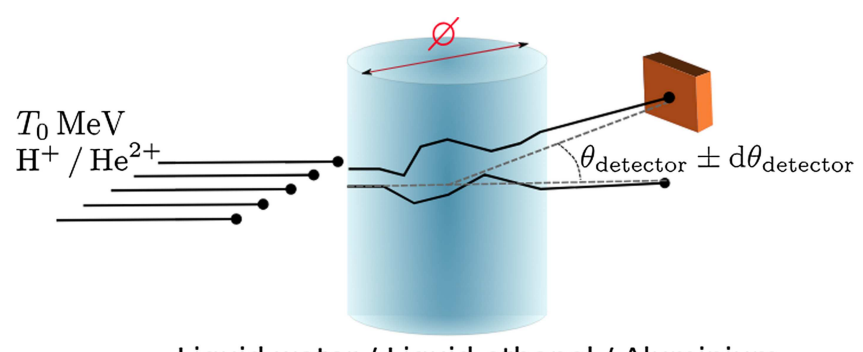

Liquid water / Liquid ethanol / Aluminium

Fig. 1. Set-up of the experiments with cylindrical targets developed by the Kyoto group [14-17,28,29]. A proton (or alpha particle) beam with an initial energy $T_{0}$ and with a width equal to the cylinder diameter $\phi$ enters the target (liquid water, liquid ethanol, or aluminium wire, depending on the experiment), and a detector analyses the energy of the particles exiting the cylinder with a given angle $\theta_{\text {detector }} \pm \mathrm{d} \theta_{\text {detector }}$.

was reported where a proton beam interacts with a liquid water jet, which was used to determine (indirectly) the stopping power of liquid water [14-17].

The experiment carried out by the group of Kyoto consisted in the irradiation of a liquid water jet with a proton beam, and the subsequent measurement of the energy distribution of the protons leaving the jet at different angles with respect to the initial beam direction. A sketch of this experimental setup is shown in Figure 1. In detail, a liquid water jet of micrometric dimensions was delivered into a vacuum chamber by a super-fine nozzle, at an appropriate flow to keep the low pressure of the vacuum chamber and the stability of the jet. Then, a swift proton beam of $2 \mathrm{MeV}$ was delivered from a Van de Graaff source, and collimated to match the dimensions of the jet, so unscattered particles do not arrive to the detector. The beam reached the jet at a point where the temperature is estimated to be $0^{\circ} \mathrm{C}$ and the density is almost $1 \mathrm{~g} / \mathrm{cm}^{3}[15]$. Then, a movable detector was placed at the other end of the jet, allowing to measure the energy distributions of the outgoing protons at different scattering

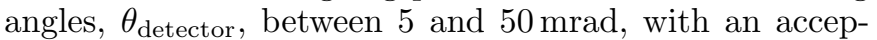
tance angle of $\mathrm{d} \theta_{\text {detector }}= \pm 2 \mathrm{mrad}$. The same system was adapted for other targets different from liquid water, so measurements for protons of $2 \mathrm{MeV}$ in an aluminium wire [16] and for protons and alpha particles of $2 \mathrm{MeV} / \mathrm{u}$ in a liquid ethanol jet $[28,29]$ were also performed.

In these experiments the exact diameter of the jet is unknown, although it should be close to the nominal nozzle diameter $(50 \mu \mathrm{m}$ for liquid water, $20 \mu \mathrm{m}$ for liquid ethanol and $26 \mu \mathrm{m}$ for the aluminium wire). Since the diameter is not exactly known, and because the experiments where intended to obtain the "unknown" values of the stopping power by their analysis by means of MC simulations, two fitting parameters where chosen by the authors in their simulations [14-17,28,29]: the jet diameter, $\phi$, and a multiplicative factor $\alpha$ to the stopping power $S_{\text {SRIM }}$ tabulated by SRIM2003, that is, $S_{\exp }\left(T_{0}\right)=$ $\alpha S_{\text {SRIM }}\left(T_{0}\right)$, for every projectile energy $T_{0}$. With these assumptions, the authors performed Monte Carlo simulations by means of the Geant4.9.1.p02 code [27], varying these two fitting parameters until the experimental projectile energy spectra were satisfactorily reproduced. The

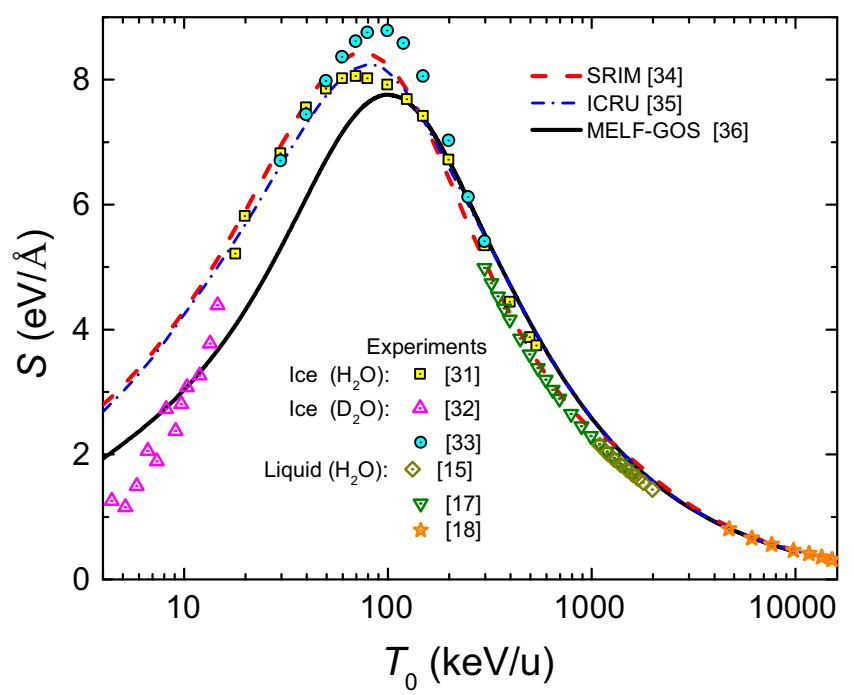

Fig. 2. Stopping power of liquid water for protons as a function of their incident energy. Symbols correspond to available experiments in $\mathrm{H}_{2} \mathrm{O}$ ice [31], $\mathrm{D}_{2} \mathrm{O}$ ice $[32,33]$ and liquid water $[15,17,18]$. Lines correspond to theoretical calculations from the dielectric formalism and the MELF-GOS methodology [36] (solid black line), from the semiempirical code SRIM2008 [34] (dashed red line), and from a compilation of data by ICRU [35] (dash-dotted blue line).

experimental results (symbols) of the projectile distribution together with the Geant4 simulations (dotted lines) will be presented in Section 5, where they are compared with our simulations obtained with the SEICS code $[8,30]$.

The stopping power of liquid water for protons indirectly determined from experiments by the Kyoto group [14-17] is shown in Figure 2, together with the experimental stopping power obtained directly by a transmission technique [18]. Due to the lack of experimental data for liquid water, we also include in the figure measurements for $\mathrm{H}_{2} \mathrm{O}$ ice [31] and $\mathrm{D}_{2} \mathrm{O}$ ice $[32,33]$. Other estimations for the stopping power of liquid water from the semiempirical SRIM code [34] or from the data compiled by ICRU [35] are also depicted. The theoretical electronic stopping power calculated by us by means of the dielectric formalism, whose methodology will be outlined in the next section, is also reported in the figure [36].

\section{Calculation of the electronic stopping power of light ions in condensed targets}

Our model to calculate the electronic stopping power of energetic ions in condensed targets is based on the dielectric formalism, taking into account the charge exchange of the projectile during its travel through the target. Also, corrections associated with the polarization of the projectile charge density are taken into account [36-38].

When energetic particles impinge on a target, most of their energy is lost by exciting its electrons, resulting in inelastic energy losses. The dielectric formalism [19], which is based on the first Born approximation, provides a simple expression for the electronic stopping power, $S$, for a 
projectile with atomic number $Z_{1}$, mass $M$ and kinetic energy $T_{0}$ (velocity $v$ ) moving through a condensed-phase material [36-38]:

$$
\begin{aligned}
S\left(T_{0}\right)= & \sum_{q=0}^{Z_{1}} \Phi_{q}\left(T_{0}\right)\left\{\frac{M e^{2}}{\pi T_{0}} \int_{0}^{\infty} \frac{\mathrm{d} k}{k}\left[Z_{1}-\rho_{q}(k)\right]^{2} \int_{0}^{k v} \mathrm{~d} \omega \omega \operatorname{Im}\left[\frac{-1}{\varepsilon(k, \omega)}\right]\right. \\
& \left.+\frac{M e^{2} Z_{1}}{\pi T_{0}} \int_{0}^{\infty} \frac{\mathrm{d} k}{k} \rho_{q} \int_{0}^{k v} \mathrm{~d} \omega \omega \operatorname{Im}\left[\frac{-1}{\varepsilon(k, \omega)}\right]\left[1-\cos \left(\omega d_{q} \sqrt{\frac{M}{2 T}}\right)\right]\right\}
\end{aligned}
$$

Here, $e$ is the absolute value of the electron charge, $\rho_{q}(k)$ is the Fourier transform of the projectile electronic density for the charge state $q, \hbar k$ and $\hbar \omega$ are, respectively, the momentum and energy transferred in an inelastic process. $\varepsilon(k, \omega)$ is the target dielectric function, and $\operatorname{Im}\left[\frac{-1}{\varepsilon(k, \omega)}\right]$ is the energy loss function (ELF) of the stopping material, which contains all the information about the possible electronic excitations in the target. In this expression, the second term denotes the contribution due to the polarization of the electronic cloud of the projectile, originated by its self-induced electric field, which displaces the centre of the projectile electronic cloud from its nucleus a distance $d_{q}[38]$.

Also, the dynamic electron capture and loss processes experienced by the projectile as it moves through the medium are included in this calculation, which results in an energy loss that depends on the projectile charge state, accounted through the probability $\Phi_{q}\left(T_{0}\right)$ of finding the projectile in the charge state $q$. These equilibrium charge states are determined through a parameterisation to experimental data [39].

At this point a good description of the target's ELF for the entire $k-\omega$ plane (i.e., of its electronic excitation spectrum) is needed. The MELF-GOS method [36,37] makes a realistic description of the electronic excitations in the condensed-phase targets, where the outer electron excitations are represented by a sum of Mermin-type ELFs [40], fitted to the experimental data in the optical limit $(k=0)$, whereas the inner-shell electron excitations are modelled by the corresponding atomic generalized oscillator strengths.

The excitation of the outer-shell electrons of the solid include both collective and single particle excitations, taking into account the finite collective excitation lifetime and its damping through phonon-assisted electronic transitions [40]. The fitting parameters at the optical limit are related with the position, width, and weight of each excitation of the experimental ELF spectrum. On the other hand, inner-shell electrons retain their atomic character, due to their large binding energies, and are described in terms of the hydrogenic generalized oscillator strengths (GOS). Also, the ELF built by the MELF-GOS methodology must fulfil physical constraints such as the $f$-sum rule at any momentum transfer. It is worth noting that using the experimental optical ELF in the calculations, all possible excitation channels are considered, including chemical bonding or physical state effects. The MELFGOS methodology has been applied successfully to several condensed-phase targets. In particular, for liquid water we have obtained excellent agreement with experimental ELF data [41] at different values of the transferred momentum [36].

In Figure 2 we present the electronic stopping power calculations of protons in liquid water based on the dielectric formalism, through equation ((1)), using the MELF-GOS model to describe the electronic excitation of the target (solid black line with the label MELF-GOS). The stopping power data compiled by ICRU [35] is shown by a dash-dotted blue line, whereas the semiempirical results provided by the SRIM code [34] are represented by a dashed red line. The predictions at high proton energies of all the models (MELF-GOS, SRIM and ICRU) agree (among them and) with the experimental data in liquid water from Siiskonen et al. [18]. At intermediate energies, around 300-2000 keV, the MELF-GOS calculations agree with the ICRU compilation, being 10\% larger than the experimental data deduced from fitting between simulations and measurements performed by the Kyoto group $[15,17]$. The SRIM data agree with the experimental data from $[15,17]$ because recently SRIM uses these experimental data for its parameterisation. It should be noted that in the SRIM curve a strange change in the slope appears at proton energies around $1 \mathrm{MeV} / \mathrm{u}$. At lower energies the predictions of the different models clearly disagree among them and depart from the available experimental data, although these are not data for liquid water but for ice. Both SRIM and ICRU curves show a better agreement with the experimental data for $\mathrm{H}_{2} \mathrm{O}$ and $\mathrm{D}_{2} \mathrm{O}$ ice [31-33], because they use a parameterisation to these experimental data. Despite the differences at low energies between the MELF-GOS predictions and the experimental data, the energies used in the simulations performed in this work are in the range $1-2 \mathrm{MeV}$ where our theoretical predictions satisfactorily agree with the experimental data.

The discrepancy in the liquid water stopping power for protons between our theoretical calculations and the experimental data from the Kyoto group [26] has stimulated us to perform simulations of its experimental setup, and to try to reproduce the projectile energy spectra after the irradiation of cylindrical targets by means of the SEICS code, where our theoretical value of the electronic stopping power is used as input data, without further refitting it.

\section{Simulation of ion transport in condensed matter}

The SEICS code (Simulation of Energetic Ions and Clusters through Solids) was designed to follow the motion of energetic ions and clusters travelling through condensed targets, which is based on a combination of Molecular Dynamics and Monte Carlo techniques [8,30]. This code includes in a detailed manner both the interaction of the projectile with the target electrons (i.e. the electronic stopping force, which is mainly responsible for the energy lost by the projectile), as well as the interaction with the target nuclei (i.e. the elastic scattering, which is mainly responsible for the beam angular spread). Given that the electronic stopping force depends on the charge-state of the projectile, the electron-capture and -loss processes 
by the projectile when it moves through the target have also been implemented in the simulation. The statistical fluctuations in the electronic energy-loss, i.e., energy-loss straggling, are also accounted for. The electronic stopping power and energy-loss straggling were obtained within the dielectric formalism and the MELF-GOS model, as it was presented in Section 3. Relativistic corrections have been introduced in the equation of motion of the projectile to properly reproduce the motion of very high kinetic energies as well as nuclear fragmentation reactions [42]. A more detailed description of the SEICS code can be found in $[8,30]$, and here only the main features will be briefly explained.

Taking into account all the previous processes, the SEICS code dynamically follows the motion of each projectile through the condensed target, providing its position, velocity and charge-state at any instant by solving its equation of motion at discrete time intervals, by means of the velocity variant of Verlet's algorithm. The force that acts on the projectile is only due to the inelastic collisions of each ion with the target electrons, which produces the so-called electronic stopping force, depending on the projectile charge-state and its velocity. To take into account the fluctuations in the force felt by the projectile, due to the stochastic nature of the interactions with the target electrons, in the simulation we get the modulus of the electronic stopping force felt by the projectile (with a given charge state) from a Gaussian distribution where its mean value is the electronic stopping power and the standard deviation is related with the energy-loss straggling [8], which are calculated from the dielectric formalism as has been outlined in the previous section. The elastic scattering between the projectile and the target atoms is described by the screened Coulomb universal interatomic potential [34].

The SEICS code has been applied successfully to study the motion of molecular ions when traversing thin layers which have applications in microelectronics or in nuclear fusion induction by inertial confinement [38]. Lately, the SEICS code has been used to simulate several problems of interest in ion beam cancer therapy, such as the evaluation of the Bragg curves as well as the lateral aperture of the beam for proton beams at therapeutic energies $[42,43]$.

\section{Results and discussion}

The SEICS code has been applied to simulate the experiment developed by the Kyoto group [15], from which the stopping power of liquid water for swift protons in the energy range $0.30-2 \mathrm{MeV}$ was deduced though a fitting procedure. As we presented in Section 2, a proton (or alpha particle) beam irradiated micrometric cylindrical targets and the energy spectra at given angles was recorded. The set-up for the simulations is the same as for the experiments (see Fig. 1), where the geometry of the jet and the position of the detector are emulated. Since the elastic peak was subtracted in the experiments, for the simulations we can assume that the width of the beam is equal to the jet diameter.

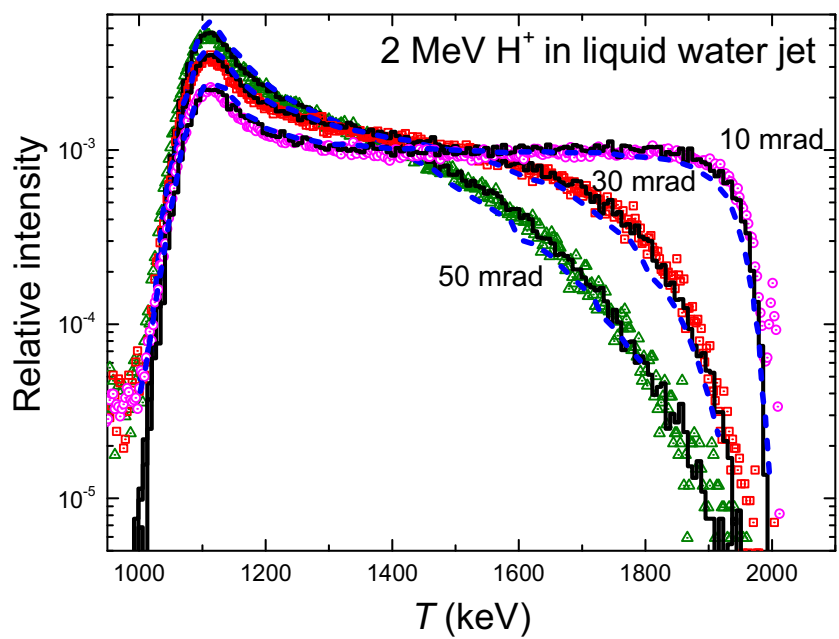

Fig. 3. Energy distribution of protons at 10, 30 and $50 \mathrm{mrad}$ after a $2 \mathrm{MeV}$ proton beam crosses a liquid water jet exiting a nozzle with a (nominal) diameter of $50 \mu \mathrm{m}$. Symbols correspond to experimental measurements from Shimizu et al. [17]. The blue dotted lines are the energy distributions obtained with the Geant4 code [17]. The solid lines represent the simulations obtained by means of the SEICS code $[8,30]$.

This case study is of particular relevance, since it offers both the possibility of benchmarking our simulation code SEICS as well as the electronic stopping power calculated with the dielectric formalism and the MELF-GOS technique. In our simulations we use only one fitting parameter, the diameter of the cylindrical target, which is not exactly known in the experiments. So, by (slightly) changing the diameters it is possible to reproduce the experimental energy spectra of protons and alpha particles after traversing cylindrical targets made of liquid water, liquid ethanol and aluminium.

In Figure 3, we display the energy distribution of a $2 \mathrm{MeV}$ proton beam irradiating a liquid water jet exiting a nozzle with a (nominal) diameter of $50 \mu \mathrm{m}$. The scattered protons have been collected for several positions of the detector: 10, 30 and $50 \mathrm{mrad}$ angles from the incident beam with $\pm 2 \mathrm{mrad}$ acceptance. The symbols are the experimental measurements from Shimizu et al. [17], obtained from the yields in their solid-state detectors (without mentioning possible effects due to energy deposition on them, which could affect a proper energy calibration). The dotted blue lines are the energy distributions obtained with the simulation code Geant4 [27], where both the diameter of the jet target and a multiplicative factor $\alpha$ to the stopping power provided by the SRIM2003 code for water vapour was used as fitting parameters to reproduce the experimental spectra (see Sect. 2). The two fitting parameters correspond to a diameter to $51 \mu \mathrm{m}$ and a parameter $\alpha=0.89$ [17]. All (experimental and simulated) curves have been scaled, in this and in subsequent figures, so they integrate to unit area.

The solid black lines represent the simulations obtained by the SEICS code, where only the diameter of the jet target was treated as a fitting parameter; neither the resolution of the detectors nor their possible dead layer effects 
were taken into account in the simulations. As it can be seen, the agreement between simulations and the experimental data is almost perfect by setting the jet diameter to $48.25 \mu \mathrm{m}$, i.e., reducing it by $3.5 \%$. Our simulated energy distribution agrees very nicely with the measurements at all the detector angles, and we obtain better results than with the Geant 4 simulations, especially at larger angles of the detector; possible background effects, as seen in the experimental data below $1 \mathrm{MeV}$, have not been incorporated in our simulations. It is worth to consider that the jet diameter can either decrease or increase. In a similar study, the actual diameter of the jet was measured and it was essentially equal to the nozzle diameter [44]. Nonetheless, it is also expected that a liquid jet evaporates in vacuum [45], thus being its diameter reduced. Taking this into account, evaporation is a plausible explanation for the $3.5 \%$ reduction in the liquid water jet diameter, although it can also be due to uncertainties in the nozzle diameter.

From the above discussion we conclude that our theoretically calculated electronic stopping power of liquid water for protons is compatible with the energy-loss measurements performed in cylindrical jets by the Kyoto group [17]. However, we estimate that the stopping power values they derive, on the basis of using two fitting parameters in their simulations (the scaling factor for the stopping power together with the jet diameter) are $10 \%$ lower than our estimate, which is in agreement with other models and experiments at larger energies [26].

In order to verify these simulation results, we have tried to reproduce the rest of the experiments performed by the Kyoto group in cylindrical targets. In particular, the bombardment of an aluminium wire with a proton beam is especially relevant and can be used as a reference, since in this case there is no possibility of evaporation, and the uncertainties in the wire diameter should be small.

In Figure 4 we show, by symbols, the experimental measurements of the proton energy distributions of $2 \mathrm{MeV}$ protons interacting with a cylindrical wire of aluminium for three positions 10, 30 and $50 \mathrm{mrad}$ of the proton detector with respect the incident proton beam. The acceptance angle is $\pm 2 \mathrm{mrad}$. The nominal diameter of the $\mathrm{Al}$ wire is $26 \mu \mathrm{m}$. The dotted blue lines are the results obtained by the Geant 4 code [16], where two fitting parameters are used (the diameter of the target and the stopping power). The solid black lines correspond to the energy spectra simulated by the SEICS code, after fitting only the wire diameter to $25.5 \mu \mathrm{m}$. In this case, there is no adjustment of the stopping power of aluminium for protons, which is provided by the dielectric formalism and the MELF-GOS method, showing a very good agreement with the abundant experimental data [46]. The experimental spectra are rather well reproduced by the SEICS simulations, with only small differences, which could be attributed to the non-perfect cylindrical shape of the wire [16]. Therefore, we have demonstrated that it is possible to reproduce the experimental measurements of the energy distributions of projectiles after traversing cylindrical aluminium targets by only using the diameter as a fitting parameter, being necessary a reduction of the wire diameter to $25.5 \mu \mathrm{m}$, that is, less than $2 \%$ of its nominal value. There is a

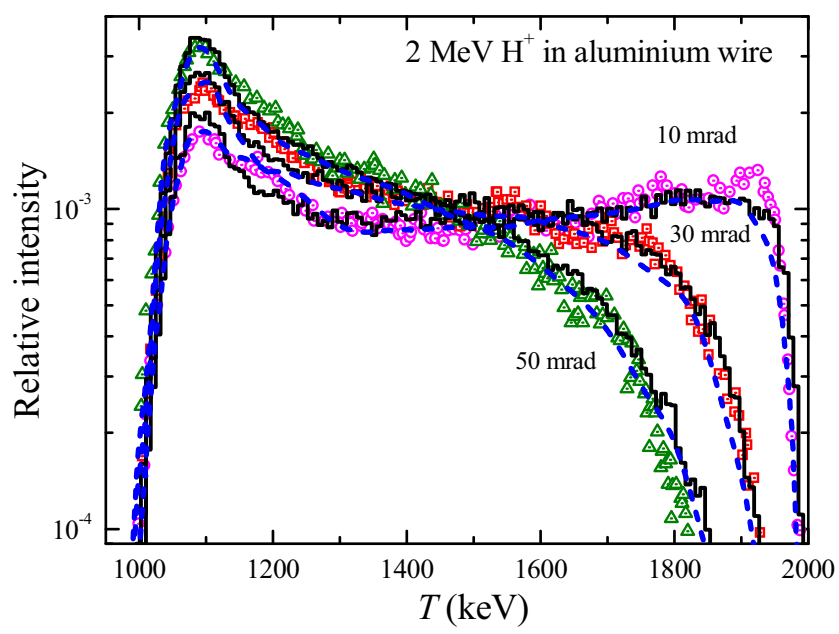

Fig. 4. Energy distribution of protons after a $2 \mathrm{MeV}$ proton beam crosses an aluminium wire with a (nominal) diameter of $26 \mu \mathrm{m}$ at three detector angles: 10, 30 and $50 \mathrm{mrad}$. Symbols correspond to experimental measurements [16]. Dotted blue lines correspond to the distributions obtained with the Geant4 code [16]. Solid black lines are the simulation obtained by the SEICS code.

very small change in the diameter in this case, as it was expected.

Finally, the last experiment performed by the Kyoto group was the measurement of the energy distributions of ions that traverse a liquid ethanol jet exiting at several angles to the detector. These results are depicted in Figure 5 by symbols. The projectiles are (a) $\mathrm{H}^{+}$and (b) $\mathrm{He}^{+2}$ with an initial energy of $2 \mathrm{MeV} / \mathrm{u}[16,28,29]$. The nominal diameter of the jet is $20 \mu \mathrm{m}$; the acceptance angle of the detector is \pm 2 mrad for $\mathrm{H}^{+}$, and $\pm 1 \mathrm{mrad}$ for $\mathrm{He}^{+2}$. These experiments are very interesting because they are reported for the irradiation with both protons and alpha particles, so a proper simulation of the energy spectra for both ions should yield the same diameter of the ethanol jet. However the simulations obtained by means of the Geant4 code, shown as dotted blue lines in the Figure 5, were obtained by fitting the stopping power and setting the jet diameter to $17.3 \mu \mathrm{m}$ for protons and to $18.4 \mu \mathrm{m}$ for alpha particles, being these results not consistent among them.

As we have commented previously, to run the SEICS code we need to know the stopping power of $\mathrm{H}^{+}$and $\mathrm{He}^{+2}$ in liquid ethanol, and in order to apply equation ((1)), we need to know the experimental optical ELF of liquid ethanol, which is not available. Therefore, in this case we will use an empirical parametric approach for predicting the optical ELF of bio-organic materials developed by Tan and co-workers [47]. Taking into account that most of the organic materials evaluated have a similar excitation spectrum with a maximum around $20 \mathrm{eV}$, they proposed that the experimental optical ELF of the outermost electrons of organic compounds can be parameterised with a single Drude-type function, with parameters that are obtained as a function of the mean atomic number of the target (i.e., the number of electrons per formula divided by the number of atoms) [47]. This approach has been applied to 

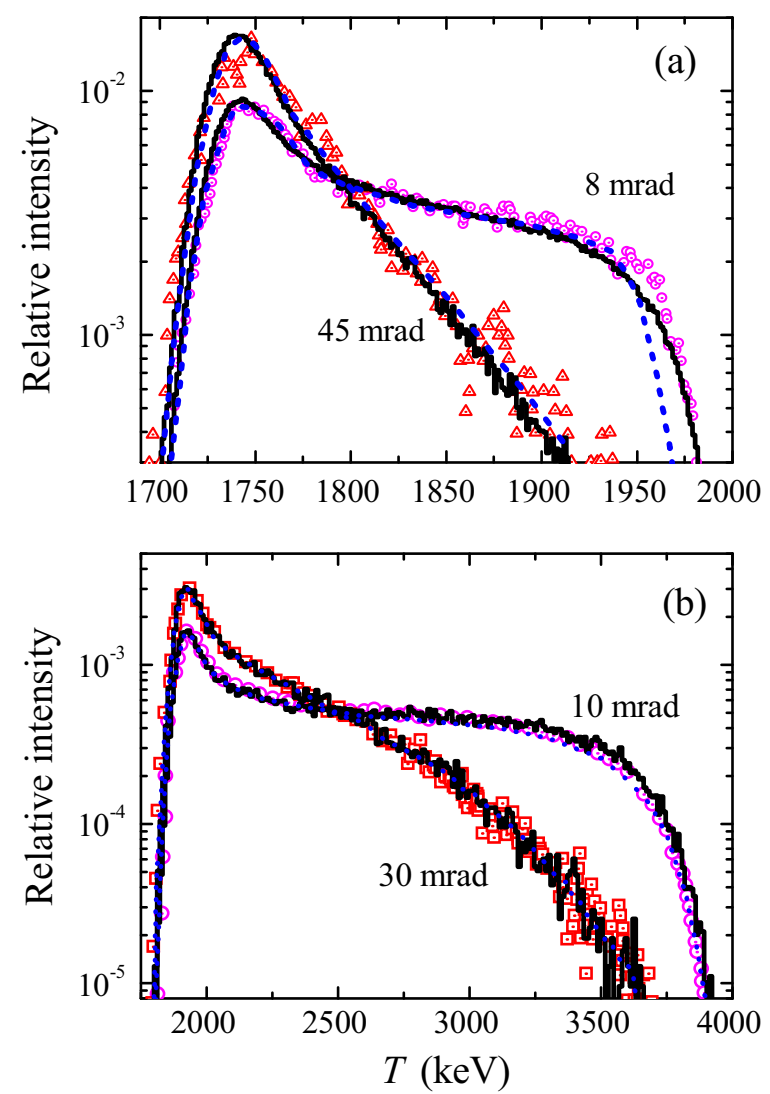

Fig. 5. Energy distribution at different angles of the detector for (a) of $2 \mathrm{MeV} \mathrm{H}^{+}$and (b) $4 \mathrm{MeV} \mathrm{He}{ }^{+2}$ beams interacting with a liquid ethanol jet with a (nominal) diameter of $20 \mu \mathrm{m}$. Symbols correspond to experimental measurements $[28,29]$. Dotted blue lines represent the simulation with the Gean4 code $[28,29]$ and solid black lines are the results obtained by the SEICS code.

obtain electronic cross sections and stopping power of relevant biological materials for different ions $[9,11,48]$. Once the optical ELF of liquid ethanol is obtained, we applied the MELF-GOS method to find the Bethe surface, which allows calculating quantities such as the electronic stopping power and the energy-loss straggling.

In Figure 6, we display by solid black lines the calculated electronic stopping power of liquid ethanol for protons and alpha particles using the dielectric formalism and the MELF-GOS method, where an optical ELF predicted by the empirical parametric approach has been used [47]. Experimental data are only available for alpha particles (triangles from [50], squares from [51] and circles from [29]) and, in this case, the comparison with the calculations is quite good, with the absolute value of the maximum fairly reproducing the results by [51], although slightly shifted in energy. Our results are also compared with the semiempirical predictions of SRIM [34] (dashed red lines), showing a very good agreement at high energies, although differences emerge around the maximum and for lower energies, where our calculations seem to be closer to the experimental data. Once the stopping quantities have been calculated, we have performed simulations of the energy spectra of protons and alpha particles in liquid

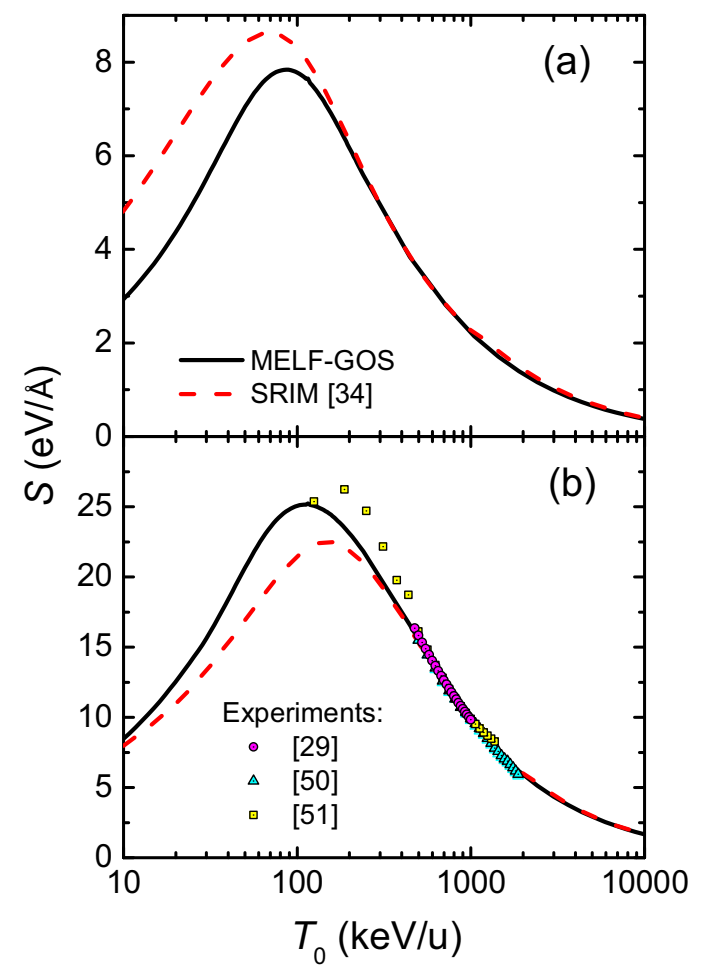

Fig. 6. Stopping power in liquid ethanol for (a) protons and (b) alpha particles. Symbols represent experimental data taken from Helmut Paul's database [49], dashed redlines are the results of SRIM [34] and solid lines are our calculations using the dielectric formalism and the MELF-GOS model with the optical ELF predicted by the parametric approach by Tan et al. [47].

ethanol. The results of our simulations are shown by solid black lines in Figure 5. The good agreement between the experiments and the simulations from the SEICS code is obtained when reducing the diameter to $18.2 \mu \mathrm{m}$ for both projectiles $\left(\mathrm{H}^{+}\right.$and $\left.\mathrm{He}^{+2}\right)$. It has to be noted that the reduction of the nominal diameter of the nozzle is $9 \%$, much larger than in the cases analysed previously of liquid water or aluminium. This fact reinforces the hypothesis of the liquid jet evaporation in vacuum, since ethanol is much more volatile than liquid water. Importantly, the energy spectra obtained by simulations with the SEICS code, without adjustment of the stopping power of liquid ethanol but using the electronic stopping power provided by the dielectric formalism, reproduce very well the experimental data for both ions. The same reduction of the jet diameter is necessary for both proton and alpha particle beams. Nevertheless, the Kyoto group [28,29] has used different diameters depending on the projectile. Therefore, it is clear that it is not necessary to use two fitting parameters to reproduce the jet experiments.

In view of the good agreement between the measurement of the energy distributions of proton and alpha particle beams after traversing cylindrical targets (water and ethanol liquid jets, and aluminium wire) with the simulations made with the SEICS code, where only the diameter of the target was treated as a free parameter, we validate our calculated electronic stopping powers obtained within 
the dielectric formalism and the MELF-GOS method, at least in the energy range covered by these experiments. Our confidence on the model is based on the suitability of the dielectric formalism for calculating electronic stopping quantities in condensed targets in this energy range, in the good agreement of the MELF-GOS ELF with the experimental Bethe surface of liquid water [36], and in the excellent agreement of our calculated electronic stopping power with the more recent experiments for liquid water at larger energies [18], as can be seen in Figure 2. Also, the mean excitation energy for liquid water predicted by the MELF-GOS method, $I=79.4 \mathrm{eV}$, is within the recommendation recently given by [25], $I=79.2 \pm 1.6 \mathrm{eV}$. In the study of the energy distributions of protons in an aluminium wire, the good agreement of our simulations with the experimental data, as well as of the calculated electronic stopping power with the large abundant experimental data [46], validate the simulations by the SEICS code.

\section{Conclusions}

Monte Carlo simulations of the transport of light ions in cylindrical condensed-phase targets (liquid water and ethanol jets, as well as aluminium wire), based on the SEICS code, have been performed in order to reproduce the experiments reported by the Kyoto group for protons and alpha particles for different detection angles [14-17]. In our simulations, the only free parameter is the diameter of the target, since we use as input reliable stopping powers for these ions calculated within the dielectric formalism by using a proper description of the Bethe surface of the materials. This is in contrast with the Geant4 simulations originally performed by the authors of the experiments, where both the jet diameter and the stopping power were used as fitting parameters [14-17]. In all the analysed cases, our simulations reproduce perfectly the full experimental set of energy distributions of projectiles measured by the Kyoto group.

In the case of liquid water, which is the most interesting target due to its ubiquity in biological environments, the jet diameter only had to be reduced by $3.5 \%$ in order to fully reproduce the experimental data for the different detection angles. This reduction is compatible with the experimental uncertainty on the jet nozzle diameter, or to the potential evaporation of the liquid jet in the vacuum chamber.

Less uncertainty is expected in the nominal diameter for the case of the aluminium wire. Here, a reduction of only $2 \%$ was needed to reproduce the experimental data. For the last jet target, liquid ethanol, a larger reduction in the jet diameter had to be introduced. However, this reduction was the same for protons and alpha particle beams. This coincidence, together with the larger volatility of ethanol as compared to liquid water, supports the obtained results.

The presented results demonstrate that it is not needed to use a scaling factor for the stopping power as a fitting parameter (together with the jet diameter, as done by the Kyoto group) in order to reproduce all the sets of experimental data for the different ions, targets and detection angles. Indeed, fixing the stopping power to an accurately calculated value makes possible to use the jet diameter as the only fitting parameter, excellently reproducing all the experimental data. The variation in the jet diameter with respect to the nominal value is minimum for the aluminium wire, where the uncertainty is expected to be less, and is larger for ethanol, which is more volatile than liquid water. This supports the hypothesis of evaporation to justify the deviation from the nominal diameter of the nozzle, and validates our Monte Carlo simulations and the electronic stopping powers calculated from the dielectric formalism, at least for ion energies larger than $100 \mathrm{keV} / \mathrm{u}$ (at which the dielectric formalism is better justified) and in the range covered by the experiments analyzed.

It should be noted that the dielectric formalism has been shown to be very useful to accurately obtain many quantities (not only the stopping power) which are needed as input for Monte Carlo simulation codes, such as electronic excitation and ionization cross sections, both for incident ions and electrons $[9,11,13]$. The stopping power is an important quantity for treatment planning (i.e., range calculation), while other cross sections (excitation, ionization) are needed to assess the effects of radiation on the nanoscale in order to estimate DNA damage. Thus, the validation of the reliability of the electronic stopping power obtained from the dielectric formalism for different condensed-phase materials serves as a further benchmark of this methodology, which is so versatile for obtaining the input data needed for track-structure simulations for applications in medical physics and nanodosimetry.

Financial support was provided by the Spanish Ministerio de Economía y Competitividad, the Spanish Ministerio de Ciencia, Innovación y Universidades and the European Regional Development Fund (Projects No. FIS2014-58849-P and PGC2018-096788-B-I00), as well as by the Fundación Séneca - Agencia de Ciencia y Tecnología de la Región de Murcia (Project No. 19907/GERM/15). PdV also acknowledges financial support provided by the Alexander von Humboldt Stiftung/Foundation through a postdoctoral fellowship.

\section{Author contribution statement}

The original idea to start this investigation was from RGM and IA. PdV and IA performed the Monte Carlo simulations with the SEICS code, which has been developed over the years by the group of RGM and IA. All authors contributed equally to analysing the results, drawing the conclusions and writing and revising the manuscript.

\section{References}

1. M. Durante, H. Paganetti, Rep. Prog. Phys. 79, 096702 (2016)

2. A. V. Solov'yov, Nanoscale Insights into Ion-beam Cancer Therapy (Springer International Publishing, Switzerland, 2017)

3. J.C. Chancellor, R.S. Blue, K.A. Cengel, S.M. AuñónChancellor, K.H. Rubins, H.G. Katzgraber, A.R. Kennedy, Microgravity, 4, 1 (2018) 
4. E. Surdutovich, A.V. Solov'yov, Eur. Phys. J. D 68, 353 (2014)

5. A. Verkhovtsev, E. Surdutovich, A.V. Solov'yov, Sci. Rep. 6, 27654 (2016)

6. H. Nikjoo, S. Uehara, D. Emfietzoglou, Interaction of Radiation with Matter (CRC Press, Boca Raton, 2012)

7. D. Emfietzoglou, H. Nikjoo, Rad. Res. 163, 98 (2005)

8. R. Garcia-Molina, I. Abril, S. Heredia-Avalos, I. Kyriakou, D. Emfietzoglou, Phys. Med. Biol. 56, 6475 (2011)

9. P. de Vera, R. Garcia-Molina, I. Abril, A.V. Solov'yov, Phys. Rev. Lett. 110, 148104 (2013)

10. M. Dingfelder, Appl. Radiat. Isot. 83 Pt B, 142 (2014)

11. P. de Vera, R. Garcia-Molina, I. Abril, Phys. Rev. Lett. 114, 018101 (2015)

12. D. Emfietzoglou, G. Papamichael, H. Nikjoo, Rad. Res. 188, 355 (2017)

13. P. de Vera, R. Garcia-Molina, J. Phys. Chem. C 123, 2075 (2019)

14. A. Itoh, M. Kaneda, S. Satoh, K. Ishii, H. Tsuchida, Nucl. Instrum. Meth. Phys. Res. B 245, 76 (2006)

15. M. Shimizu, M. Kaneda, T. Hayakawa, H. Tsuchida, A. Itoh, Nucl. Instr. Meth. Phys. Res. B 267, 2667 (2009)

16. A. Itoh, M. Kaneda, M. Shimizu, T. Hayakawa, T. Iriki, H. Tsuchida, Vacuum 84, 999 (2010)

17. M. Shimizu, T. Hayakawa, M. Kaneda, H. Tsuchida, A. Itoh, Vacuum 84, 1002 (2010)

18. T. Siiskonen, H. Kettunen, K. Peräjärvi, A. Javanainen, M. Rossi, W.H. Trzaska, J. Turunen, A. Virtanen, Phys. Med. Biol. 56, 2367 (2011)

19. J. Lindhard, K. Dan. Vidensk. Selsk. Mat.-Fys. Medd. 28, 8 (1954)

20. M. Dingfelder, M. Inokuti, H.G. Paretzke, Radiat. Phys. Chem. 59, 255 (2000)

21. M. Dingfelder, Radiat. Protect. Dosim. 99, 23 (2002)

22. D. Emfietzoglou, M. Moscovitch, Nucl. Instrum. Meth. Phys. Res. B 209, 239 (2003)

23. D. Emfietzoglou, Radiat. Phys. Chem. 66, 373 (2003)

24. A. Besemer, H. Paganetti, B. Bednarz, Phys. Med. Biol. 58, 887 (2013)

25. H. Paul, Adv. Quantum Chem. 65, 39 (2013)

26. R. Garcia-Molina, I. Abril, P. de Vera, H. Paul, Nucl. Instrum. Meth. Phys. Res. B 299, 51 (2013)

27. Geant4, 2008. Available from http://geant4.web.cern. ch/geant4/

28. M. Kaneda, S. Sato, M. Shimizu, Z. He, K. Ishii, H. Tsuchida, A. Itoh, Nucl. Instrum. Meth. Phys. Res. B 256, $97(2007)$

29. M. Shimizu, T. Hayakawa, K. Hisano, M. Kaneda, H. Tsuchida, A. Itoh, Nucl. Instrum. Meth. Phys. Res. B 269, 810 (2011)
30. R. Garcia-Molina, I. Abril, P. de Vera, I. Kyriakou, D. Emfietzoglou, Chap. 8 in Fast ion-atom and ionmoleculecollisions, edited by D. Belkic (World Scientific Publishing Company, Singapore, 2012)

31. P. Bauer, W. Käferböck, V. Nečas, Nucl. Instrum. Meth. Phys. Res. B 93, 132 (1994)

32. W.A. Wenzel, W. Whaling, Phys. Rev. 87, 499 (1952)

33. D.A. Andrews, G. Newton, J. Phys. D 10, 845 (1977)

34. J.F. Ziegler, J.P. Biersack, M.D. Ziegler, SRIM - The Stopping and Range of Ions in Matter (SRIM Co., Chester, Maryland, 2008)

35. ICRU, Report 49 - Stopping Powers and Ranges for Protons and Alpha Particles, (International Commission on Radiation Units and Measurements, Bethesda, Maryland, 1993)

36. I. Abril, R. Garcia-Molina, P. de Vera, I. Kyriakou, D. Emfietzoglou, Adv. Quantum Chem. 65, 129 (2013)

37. S. Heredia-Avalos, R. Garcia-Molina, J.M. FernándezVarea, I. Abril, Phys. Rev. A 72, 052902 (2005)

38. S. Heredia-Avalos, I. Abril, C.D. Denton, J.C. MorenoMarín, R. Garcia-Molina, J. Phys. Cond. Matter 19, 466205 (2007)

39. P.L. Grande, G. Schiwietz, CasP - Convolution approximation for swift particles, version 3.1 (2005) code available at http://www.hmi.de/people/schiwietz/casp.html

40. N.D. Mermin, Phys. Rev. B 1, 2362 (1970)

41. H. Hayashi, N. Watanabe, Y. Udagawa, C.-C. Kao, J. Proc. Nat. Acad. Sci. 97, 6264 (2000)

42. P. de Vera, I. Abril, R. Garcia-Molina, Rad. Res. 190, 282 (2018)

43. I. Abril, P. de Vera, R. Garcia-Molina, I. Kyriakou, D. Emfietzoglou, Nucl. Instrum. Meth. Phys. Res. B 352, 176 (2015)

44. T. Kondow, F. Mafuné, Ann. Rev. Phys. Chem. 51, 731 (2000)

45. M. Faubel, S. Schlemmer, J. P. Toennies, Z. Phys. D 10, 269 (1988)

46. C.D. Denton, I. Abril, J.C. Moreno-Marín, S. HerediaAvalos, R. Garcia-Molina, Phys. Stat. Solidi. B 245, 1498 (2008)

47. Z. Tan, Y. Xia, M. Zhao, X. Liu, F. Li, B. Huang, Y. Ji, Nucl. Instrum. Meth. Phys. Res. B 222, 27 (2004)

48. S. Limandri, P. de Vera, R.C. Fadanelli, L.C.C.M. Nagamine, A. Mello, R. Garcia-Molina, M. Behar, I. Abril, Phys. Rev. E 89, 022703 (2014)

49. H. Paul, Stopping Power of Matter for Ions. Graphs, Data, Comments and Programs, https://www-nds.iaea.org/ stopping/

50. A. Akhavan-Rezayat, R.B.J. Palmer, J. Phys. E 13, 877 (1980)

51. A.K.M.M. Haque, A. Mohammadi, H. Nikjoo, Radiat. Protect. Dosim. 13, 71 (1985) 